\title{
On Oscillatory and Asymptotic Behavior of a Second-Order Nonlinear Damped Neutral Differential Equation
}

\author{
Ercan Tunç ${ }^{1}$ and Said R. Grace ${ }^{2}$ \\ ${ }^{1}$ Department of Mathematics, Faculty of Arts and Sciences, Gaziosmanpaşa University, 60240 Tokat, Turkey \\ ${ }^{2}$ Department of Engineering Mathematics, Faculty of Engineering, Cairo University, Orman, Giza 12221, Egypt \\ Correspondence should be addressed to Ercan Tunç; ercantunc72@yahoo.com
}

Received 14 April 2016; Revised 30 July 2016; Accepted 9 August 2016

Academic Editor: Davood D. Ganji

Copyright (c) 2016 E. Tunç and S. R. Grace. This is an open access article distributed under the Creative Commons Attribution License, which permits unrestricted use, distribution, and reproduction in any medium, provided the original work is properly cited.

This paper discusses oscillatory and asymptotic properties of solutions of a class of second-order nonlinear damped neutral differential equations. Some new sufficient conditions for any solution of the equation to be oscillatory or to converge to zero are given. The results obtained extend and improve some of the related results reported in the literature. The results are illustrated with examples.

\section{Introduction}

In this paper, we consider the second-order nonlinear damped neutral differential equation

$$
\begin{aligned}
& \left(r(t)\left(z^{\prime}(t)\right)^{\alpha}\right)^{\prime}+h(t)\left(z^{\prime}(t)\right)^{\alpha}+q(t) f(x(\delta(t))) \\
& \quad=0, \quad t \geq t_{0}>0
\end{aligned}
$$

where $z(t)=x(t)-p(t) x(\tau(t))$ and $\alpha$ is a ratio of positive odd integers. Throughout this paper and without further mention, we assume that

(i) $p:\left[t_{0}, \infty\right) \rightarrow \mathbb{R}$ is a real-valued continuous function with $0 \leq p(t) \leq p_{0}<1$;

(ii) $h:\left[t_{0}, \infty\right) \rightarrow \mathbb{R}$ and $q:\left[t_{0}, \infty\right) \rightarrow[0, \infty)$ are realvalued continuous functions, and $q(t)$ is not identically zero for all sufficiently large $t$;

(iii) $r:\left[t_{0}, \infty\right) \rightarrow \mathbb{R}^{+}=(0, \infty)$ is a real-valued continuous function such that

$$
R\left(t, t_{0}\right):=\int_{t_{0}}^{t} \frac{d s}{(\gamma(s) r(s))^{1 / \alpha}} \longrightarrow \infty \quad \text { as } t \longrightarrow \infty,
$$

where

$$
\gamma(t)=\exp \left(\int_{t_{0}}^{t} \frac{h(s)}{r(s)} d s\right) ;
$$

(iv) $f: \mathbb{R} \rightarrow \mathbb{R}$ is a real-valued continuous function with $u f(u)>0$ for $u \neq 0$ and there exists a constant $k>0$ such that $f(u) / u^{\beta} \geq k$ for all $u \neq 0$, where $\beta$ is a ratio of positive odd integers with $\alpha \geq \beta$;

(v) $\tau, \delta:\left[t_{0}, \infty\right) \rightarrow \mathbb{R}$ are real-valued continuous functions such that $\tau(t) \leq t, \lim _{t \rightarrow \infty} \tau(t)=\infty$, and $\lim _{t \rightarrow \infty} \delta(t)=\infty$.

Without further mention, we will assume throughout that every solution $x(t)$ of (1) that is under consideration here is continuable to the right and nontrivial; that is, $x(t)$ is defined on some ray $\left[t_{x}, \infty\right)$ for some $t_{x} \geq t_{0}$ and $\sup \{|x(t)|: t \geq$ $\left.t_{1}\right\}>0$ for every $t_{1} \geq t_{x}$, which has the properties $z(t) \epsilon$ $C^{1}\left(\left[t_{x}, \infty\right), \mathbb{R}\right)$ and $r(t)\left(z^{\prime}(t)\right)^{\alpha} \in C^{1}\left(\left[t_{x}, \infty\right), \mathbb{R}\right)$ for any $t_{x} \geq$ $t_{0}$. We tacitly assume that (1) possesses such solutions. Such a solution is said to be oscillatory if it has arbitrarily large zeros on $\left[t_{x}, \infty\right)$; otherwise, it is called nonoscillatory. Equation (1) is said to be oscillatory if all of its solutions are oscillatory.

Since Sturm [1] introduced the concept of oscillation when he studied the problem of the heat transmission, 
the oscillation theory has been a very active area of research in the qualitative theory of both ordinary and functional differential equations. Usually, a qualitative approach is concerned with the behavior of solutions of a given differential equation and does not seek explicit solutions. Since then, asymptotic and oscillatory properties of solutions to different classes of ordinary differential equations, functional differential equations, and dynamic equations have attracted the attention of many researchers; see, for example, [2-27] and the references therein (see also [28-33] for numerical methods with semianalytical methods).

Recently, neutral delay differential equations, that is, equations in which the highest order derivative of the unknown function appears both with and without delays, have received strong interest in the study of oscillation properties of their solutions. The problem of asymptotic and oscillatory behavior or solutions of neutral differential equations is of both theoretical and practical interest. One reason for this is that they arise, for example, in applications to electric networks containing lossless transmission lines. Such networks appear in high speed computers where lossless transmission lines are used to interconnect switching circuits. They also occur in problems dealing with vibrating masses attached to an elastic bar and in the solution of variational problems with time delays. Interested readers can refer to the book by Hale [34] for some applications in science and technology.

On reviewing the literature, it becomes apparent that most results concerning the oscillation of all solutions of (1) are for the special case when $h(t)=0$. Regarding the oscillation of undamped neutral differential equations, that is, special cases of (1) with $h(t)=0$, many papers have been published for different cases of $p(t)$ such as $-1 \leq p(t) \leq 0$, $-\infty<p_{0} \leq p(t) \leq 0$, and $0 \leq p(t) \leq p_{0}<1$. We refer the reader to $[4,14,16-20,24-26]$ and the references cited therein as examples of recent results on this topic.

In 2015, Li et al. [20] considered (1) with $h(t)=0$ in the case where $\delta(t) \leq t, \delta^{\prime}(t)>0$, and $\alpha=\beta$ and presented some new conditions which ensure that any solution $x$ of (1) with $h(t)=0$ either is oscillatory or converges to zero.

Motivated by the work of $\mathrm{Li}$ et al. [20] and the papers mentioned above, in the present paper, by employing Riccati type transformation and the integral averaging technique involving integrals and/or weighted integrals of coefficients of a given differential equation, we establish some new sufficient conditions for all solutions of (1) to be oscillatory or to converge to zero. The results obtained improve the results of Li et al. [20] in the sense that we do not require the restrictive condition $\delta^{\prime}(t)>0$ and extend some known results in the relevant literature. It should be noted that Li et al. [20] only discussed the oscillation properties of solutions in the delay case $\delta(t) \leq t$. Here, we also consider the advanced case $\delta(t) \geq$ $t$ as well. Some examples are also considered to illustrate the main results. We also want to note that the results obtained can easily be extended to more general neutral differential equations and neutral dynamic equations on any time scales of the type (1). It is therefore hoped that the present paper will contribute to the studies on oscillatory and asymptotic behavior of solutions of neutral differential equations with damping term.

\section{Main Results}

For any continuous function $u(t)$, we set $u_{+}(t)=\max \{0, u(t)\}$, and, to simplify the formulation of our results, we will use the following notations:

$$
R\left(t, t_{1}\right):=\int_{t_{1}}^{t} \frac{d s}{(\gamma(s) r(s))^{1 / \alpha}} \quad \text { for } t_{0} \leq t_{1} \leq t<\infty,
$$

and, for sufficiently large $t_{*}$,

$$
\begin{aligned}
& \theta\left(t, t_{*}\right):=\frac{R\left(\delta(t), t_{*}\right)}{R\left(t, t_{*}\right)}, \\
& \phi\left(t, t_{*}\right):= \begin{cases}1, & \delta(t) \geq t, \\
\theta^{\beta}\left(t, t_{*}\right), & \delta(t) \leq t .\end{cases}
\end{aligned}
$$

We begin with a lemma that will be used to prove our main results.

Lemma 1 (see [35]). If $X$ and $Y$ are nonnegative and $\lambda>1$, then

$$
\lambda X Y^{\lambda-1}-X^{\lambda} \leq(\lambda-1) Y^{\lambda}
$$

where the equality holds if and only if $X=Y$.

Theorem 2. Assume that condition (2) is satisfied and there exists a positive function $\rho \in C^{1}\left(\left[t_{0}, \infty\right), \mathbb{R}\right)$ such that, for all sufficiently large $T_{*}$ and for $T>T_{*}$,

$$
\begin{aligned}
& \limsup _{t \rightarrow \infty} \int_{T}^{t}\left\{k \rho(s) \gamma(s) q(s) \phi\left(s, T_{*}\right)-\frac{c^{*} \rho_{+}^{\prime}(s)}{R^{\beta}\left(s, T_{*}\right)}\right\} d s \\
& \quad=\infty
\end{aligned}
$$

with $c^{*}>0$; then, any solution $x$ of (1) either oscillates or tends to zero as $t \rightarrow \infty$.

Proof. Let $x(t)$ be a nonoscillatory solution of (1). Without loss of generality, we may assume that there exists $t_{1} \geq t_{0}$ such that $x(t)>0, x(\tau(t))>0$, and $x(\delta(t))>0$ for $t \geq t_{1}$. Multiplying (1) by $\gamma(t)$ (see (3)), (1) takes the form

$$
\left(\gamma(t) r(t)\left(z^{\prime}(t)\right)^{\alpha}\right)^{\prime}+q(t) \gamma(t) f(x(\delta(t)))=0 .
$$

From (ii), (3), (iv), and (8), we have

$$
\begin{array}{r}
\left(\gamma(t) r(t)\left(z^{\prime}(t)\right)^{\alpha}\right)^{\prime}=-q(t) \gamma(t) f(x(\delta(t))) \leq 0 \\
\text { for } t \geq t_{1},
\end{array}
$$

so $\gamma(t) r(t)\left(z^{\prime}(t)\right)^{\alpha}$ is eventually decreasing, say for $t \in$ $\left[t_{2}, \infty\right) \subset\left[t_{1}, \infty\right)$. We claim that

$$
z^{\prime}(t)>0 \text { for } t \geq t_{2}
$$


If this is not so, then there exists $t_{3} \in\left[t_{2}, \infty\right)$ such that $z^{\prime}\left(t_{3}\right) \leq$ 0 . In view of (9), there is $t_{4} \geq t_{3}$ such that

$$
\gamma(t) r(t)\left(z^{\prime}(t)\right)^{\alpha} \leq \gamma\left(t_{4}\right) r\left(t_{4}\right)\left(z^{\prime}\left(t_{4}\right)\right)^{\alpha}:=c<0
$$

for $t \in\left[t_{4}, \infty\right)$. Hence,

$$
z^{\prime}(t) \leq c^{1 / \alpha} \frac{1}{(\gamma(t) r(t))^{1 / \alpha}}
$$

from which it follows that

$$
z(t) \leq z\left(t_{4}\right)+c^{1 / \alpha} \int_{t_{4}}^{t} \frac{d s}{(\gamma(s) r(s))^{1 / \alpha}} \quad \text { for } t \geq t_{4} .
$$

In view of (2), (13) implies that

$$
\lim _{t \rightarrow \infty} z(t)=-\infty
$$

Thus, there are two cases to consider.

Case 1. If $x(t)$ is unbounded, then there exists a sequence $\left\{t_{k}\right\}$ such that $\lim _{k \rightarrow \infty} t_{k}=\infty$ and $\lim _{k \rightarrow \infty} x\left(t_{k}\right)=\infty$, where

$$
x\left(t_{k}\right)=\max \left\{x(s): t_{0} \leq s \leq t_{k}\right\} .
$$

Since $\lim _{t \rightarrow \infty} \tau(t)=\infty$, we can choose a large $k$ such that $\tau\left(t_{k}\right)>t_{0}$. Thus, by (15) and the fact that $\tau(t) \leq t$, we have

$$
\begin{aligned}
x\left(\tau\left(t_{k}\right)\right) & =\max \left\{x(s): t_{0} \leq s \leq \tau\left(t_{k}\right)\right\} \\
& \leq \max \left\{x(s): t_{0} \leq s \leq t_{k}\right\}=x\left(t_{k}\right),
\end{aligned}
$$

so, from the definition of $z$, we see that

$$
\begin{aligned}
z\left(t_{k}\right) & =x\left(t_{k}\right)-p\left(t_{k}\right) x\left(\tau\left(t_{k}\right)\right) \geq\left(1-p\left(t_{k}\right)\right) x\left(t_{k}\right) \\
& \geq\left(1-p_{0}\right) x\left(t_{k}\right)>0
\end{aligned}
$$

which contradicts (14).

Case 2. If $x(t)$ is bounded, then, in view of the definition of $z$ and the fact that $0 \leq p(t) \leq p_{0}<1$, it follows that $z$ is also bounded, which again contradicts (14). Thus, in view of Cases 1 and 2, we conclude that (10) holds.

Hence, from (9) and (10) and the definition of $z$, we conclude that there exists $t_{2} \geq t_{1}$ such that, for $t \geq t_{2}$, either

$$
\begin{aligned}
z(t) & >0, \\
z^{\prime}(t) & >0, \\
\left(\gamma(t) r(t)\left(z^{\prime}(t)\right)^{\alpha}\right)^{\prime} & \leq 0
\end{aligned}
$$

or

$$
\begin{aligned}
z(t) & <0, \\
z^{\prime}(t) & >0, \\
\left(\gamma(t) r(t)\left(z^{\prime}(t)\right)^{\alpha}\right)^{\prime} & \leq 0 .
\end{aligned}
$$

Assume that (18) holds. We note that $x(t) \geq z(t)$ and set

$$
w(t)=\rho(t) \frac{\gamma(t) r(t)\left(z^{\prime}(t)\right)^{\alpha}}{z^{\beta}(t)} \text { for } t \geq t_{2} .
$$

Then, $w(t)>0$ for $t \geq t_{2}$ and, from (1), (3), (iv), and (20), we obtain

$$
\begin{aligned}
& w^{\prime}(t)=\rho^{\prime}(t) \frac{\gamma(t) r(t)\left(z^{\prime}(t)\right)^{\alpha}}{z^{\beta}(t)}+\rho(t) \\
& {\left[\frac{\left(\gamma(t) r(t)\left(z^{\prime}(t)\right)^{\alpha}\right)^{\prime}}{z^{\beta}(t)}\right.} \\
& \left.-\beta \frac{\gamma(t) r(t)\left(z^{\prime}(t)\right)^{\alpha} z^{\beta-1}(t) z^{\prime}(t)}{z^{2 \beta}(t)}\right]=\rho^{\prime}(t) \\
& \frac{\gamma(t) r(t)\left(z^{\prime}(t)\right)^{\alpha}}{z^{\beta}(t)}+\rho(t) \\
& \frac{\gamma(t)\left[h(t)\left(z^{\prime}(t)\right)^{\alpha}+\left(r(t)\left(z^{\prime}(t)\right)^{\alpha}\right)^{\prime}\right]}{z^{\beta}(t)}-\beta \rho(t) \\
& \gamma(t) r(t) \frac{\left(z^{\prime}(t)\right)^{\alpha+1}}{z^{\beta+1}(t)}=\rho^{\prime}(t) \frac{\gamma(t) r(t)\left(z^{\prime}(t)\right)^{\alpha}}{z^{\beta}(t)} \\
& -\rho(t) \gamma(t) q(t) \frac{f(x(\delta(t)))}{z^{\beta}(t)}-\beta \rho(t) \gamma(t) r(t) \\
& \cdot \frac{\left(z^{\prime}(t)\right)^{\alpha+1}}{z^{\beta+1}(t)} \leq \rho_{+}^{\prime}(t) \frac{\gamma(t) r(t)\left(z^{\prime}(t)\right)^{\alpha}}{z^{\beta}(t)}-k \rho(t) \\
& \gamma(t) q(t) \frac{x^{\beta}(\delta(t))}{z^{\beta}(t)}-\beta \rho(t) \gamma(t) r(t) \frac{\left(z^{\prime}(t)\right)^{\alpha+1}}{z^{\beta+1}(t)} \\
& \leq \rho_{+}^{\prime}(t) \frac{\gamma(t) r(t)\left(z^{\prime}(t)\right)^{\alpha}}{z^{\beta}(t)}-k \rho(t) \gamma(t) q(t) \\
& \cdot \frac{z^{\beta}(\delta(t))}{z^{\beta}(t)}-\beta \rho(t) \gamma(t) r(t) \frac{\left(z^{\prime}(t)\right)^{\alpha+1}}{z^{\beta+1}(t)} \text {. }
\end{aligned}
$$

In view of the fact that $z^{\prime}(t)>0$ for $t \geq t_{2}$, it follows from (21) that

$$
\begin{aligned}
w^{\prime}(t) \leq & \rho_{+}^{\prime}(t) \gamma(t) r(t)\left(\frac{z^{\prime}(t)}{z(t)}\right)^{\alpha} z^{\alpha-\beta}(t) \\
& -k \rho(t) \gamma(t) q(t)\left(\frac{z(\delta(t))}{z(t)}\right)^{\beta} .
\end{aligned}
$$


Since $\gamma(t) r(t)\left(z^{\prime}(t)\right)^{\alpha}$ is nonincreasing, we see that

$$
\begin{aligned}
z(t) & \geq z(t)-z\left(t_{2}\right)=\int_{t_{2}}^{t} \frac{\left(\gamma(s) r(s)\left(z^{\prime}(s)\right)^{\alpha}\right)^{1 / \alpha}}{(\gamma(s) r(s))^{1 / \alpha}} d s \\
& \geq(\gamma(t) r(t))^{1 / \alpha} z^{\prime}(t) \int_{t_{2}}^{t} \frac{1}{(\gamma(s) r(s))^{1 / \alpha}} d s,
\end{aligned}
$$

from which it follows that

$$
\frac{z^{\prime}(t)}{z(t)} \leq \frac{1}{(\gamma(t) r(t))^{1 / \alpha} R\left(t, t_{2}\right)} .
$$

Using again the fact that $\gamma(t) r(t)\left(z^{\prime}(t)\right)^{\alpha}$ is eventually decreasing for $t \geq t_{2}$, we get

$$
\begin{aligned}
0<\gamma(t) r(t)\left(z^{\prime}(t)\right)^{\alpha} \leq \gamma\left(t_{2}\right) r\left(t_{2}\right)\left(z^{\prime}\left(t_{2}\right)\right)^{\alpha} & :=c_{1} \\
\forall t & \geq t_{2},
\end{aligned}
$$

and thus we have, for all $t \geq t_{3}:=t_{2}+1$, that

$$
\begin{aligned}
z(t) & \leq z\left(t_{2}\right)+c_{1}^{1 / \alpha} \int_{t_{2}}^{t} \frac{d s}{(\gamma(s) r(s))^{1 / \alpha}} \\
& =z\left(t_{2}\right)+c_{1}^{1 / \alpha} R\left(t, t_{2}\right) \\
& =\left[\frac{z\left(t_{2}\right)}{R\left(t, t_{2}\right)}+c_{1}^{1 / \alpha}\right] R\left(t, t_{2}\right) \\
& \leq\left[\frac{z\left(t_{2}\right)}{R\left(t_{3}, t_{2}\right)}+c_{1}^{1 / \alpha}\right] R\left(t, t_{2}\right)=c_{2} R\left(t, t_{2}\right)
\end{aligned}
$$

holds, where $c_{2}=\left[z\left(t_{2}\right) / R\left(t_{3}, t_{2}\right)+c_{1}^{1 / \alpha}\right]$.

Using (24) and (26) in (22), we obtain

$$
w^{\prime}(t) \leq \frac{c_{3} \rho_{+}^{\prime}(t)}{R^{\beta}\left(t, t_{2}\right)}-k \rho(t) \gamma(t) q(t)\left(\frac{z(\delta(t))}{z(t)}\right)^{\beta}
$$

where $c_{3}=c_{2}^{\alpha-\beta}$

for $t \geq t_{2}$,

Now, if $\delta(t) \geq t$, in view of the fact that $z(t)$ is increasing, we have

$$
\frac{z(\delta(t))}{z(t)} \geq 1
$$

Using (28) in (27), we get

$$
w^{\prime}(t) \leq \frac{c_{3} \rho_{+}^{\prime}(t)}{R^{\beta}\left(t, t_{2}\right)}-k \rho(t) \gamma(t) q(t) .
$$

Next, if $\delta(t) \leq t$, in view of the fact that $\lim _{t \rightarrow \infty} \delta(t)=\infty$, we can choose $t_{3}>t_{2}$ such that $\delta(t) \geq t_{2}$ for all $t \geq t_{3}$. Thus, from the fact that $\gamma(t) r(t)\left(z^{\prime}(t)\right)^{\alpha}$ is eventually decreasing, we have

$$
\begin{aligned}
& z(t)-z(\delta(t))=\int_{\delta(t)}^{t} \frac{\left(\gamma(s) r(s)\left(z^{\prime}(s)\right)^{\alpha}\right)^{1 / \alpha}}{(\gamma(s) r(s))^{1 / \alpha}} d s \\
& \leq\left(\gamma(\delta(t)) r(\delta(t))\left(z^{\prime}(\delta(t))\right)^{\alpha}\right)^{1 / \alpha} R(t, \delta(t)) ;
\end{aligned}
$$

that is,

$$
\begin{aligned}
& \frac{z(t)}{z(\delta(t))} \leq 1 \\
& \quad+\frac{\left(\gamma(\delta(t)) r(\delta(t))\left(z^{\prime}(\delta(t))\right)^{\alpha}\right)^{1 / \alpha}}{z(\delta(t))}\left[R\left(t, t_{2}\right)\right. \\
& \left.\quad-R\left(\delta(t), t_{2}\right)\right], \\
& z(\delta(t)) \geq z(\delta(t))-z\left(t_{2}\right) \\
& \quad=\int_{t_{2}}^{\delta(t)} \frac{\left(\gamma(s) r(s)\left(z^{\prime}(s)\right)^{\alpha}\right)^{1 / \alpha}}{(\gamma(s) r(s))^{1 / \alpha}} d s \\
& \quad \geq\left(\gamma(\delta(t)) r(\delta(t))\left(z^{\prime}(\delta(t))\right)^{\alpha}\right)^{1 / \alpha} R\left(\delta(t), t_{2}\right) ;
\end{aligned}
$$

that is,

$$
\begin{aligned}
& \frac{\left(\gamma(\delta(t)) r(\delta(t))\left(z^{\prime}(\delta(t))\right)^{\alpha}\right)^{1 / \alpha}}{z(\delta(t))} \leq \frac{1}{R\left(\delta(t), t_{2}\right)} \\
& \text { for } t \geq t_{3} .
\end{aligned}
$$

From (31) and (33), it is easy to see that

$$
\frac{z(\delta(t))}{z(t)} \geq \frac{R\left(\delta(t), t_{2}\right)}{R\left(t, t_{2}\right)}=\theta\left(t, t_{2}\right) \quad \text { for } t \geq t_{3} .
$$

Using (34) in (27), we find that

$$
w^{\prime}(t) \leq \frac{c_{3} \rho_{+}^{\prime}(t)}{R^{\beta}\left(t, t_{2}\right)}-k \rho(t) \gamma(t) q(t) \theta^{\beta}\left(t, t_{2}\right) .
$$

Combining (29) and (35), we see that

$$
w^{\prime}(t) \leq-k \rho(t) \gamma(t) q(t) \phi\left(t, t_{2}\right)+\frac{c_{3} \rho_{+}^{\prime}(t)}{R^{\beta}\left(t, t_{2}\right)}
$$

for $t \geq t_{3}$.

Integrating this inequality from $t_{3}$ to $t$ yields

$$
\begin{aligned}
& \int_{t_{3}}^{t}\left\{k \rho(s) \gamma(s) q(s) \phi\left(s, t_{2}\right)-\frac{c_{3} \rho_{+}^{\prime}(s)}{R^{\beta}\left(s, t_{2}\right)}\right\} d s \\
& \quad \leq w\left(t_{3}\right)-w(t)<w\left(t_{3}\right),
\end{aligned}
$$

which contradicts condition (7).

Now, let (19) hold. Then, we claim that $\lim _{t \rightarrow \infty} x(t)=0$. In view of $z(t)<0$ and $z^{\prime}(t)>0$, we have

$$
\lim _{t \rightarrow \infty} z(t)=l \leq 0,
$$

where $l$ is a constant, and so $z(t)$ is bounded for sufficiently large $t$. We assert that $x(t)$ is also bounded. Otherwise, if $x(t)$ is unbounded, then there exists a sequence $\left\{t_{k}\right\}$ such that 
$\lim _{k \rightarrow \infty} t_{k}=\infty$ and $\lim _{k \rightarrow \infty} x\left(t_{k}\right)=\infty$, where $x\left(t_{k}\right)$ is as in (15), and so, from the definition of $z$ and $\tau(t) \leq t$, we see that

$$
\begin{aligned}
z\left(t_{k}\right) & =x\left(t_{k}\right)-p\left(t_{k}\right) x\left(\tau\left(t_{k}\right)\right) \geq\left(1-p\left(t_{k}\right)\right) x\left(t_{k}\right) \\
& >0
\end{aligned}
$$

which contradicts the fact that $z(t)<0$ for $t \geq t_{2}$, and so $x(t)$ is bounded. Therefore, we have

$$
\limsup _{t \rightarrow \infty} x(t)=a, \quad 0 \leq a<\infty .
$$

If $a>0$, then there exists a sequence $\left\{t_{n}\right\}$ such that $\lim _{n \rightarrow \infty} t_{n}=\infty$ and $\lim _{n \rightarrow \infty} x\left(t_{n}\right)=a$. Let $\varepsilon=a\left(1-p_{0}\right) / 2 p_{0}$; then, for all large $n$, we have $x\left(\tau\left(t_{n}\right)\right)<a+\varepsilon$. From this and the definition of $z$, we obtain

$$
\begin{aligned}
0 & \geq \lim _{n \rightarrow \infty} z\left(t_{n}\right) \geq \lim _{n \rightarrow \infty} x\left(t_{n}\right)-p_{0}(a+\varepsilon)=\frac{a\left(1-p_{0}\right)}{2} \\
& >0
\end{aligned}
$$

which contradicts the fact that $z(t)<0$, and hence $\limsup _{t \rightarrow \infty} x(t)=0$. Now, in view of the fact that $x(t)>0$, we conclude that $\lim _{t \rightarrow \infty} x(t)=0$, which completes the proof of Theorem 2.

Theorem 3. Let (2) be satisfied. If there exists a positive function $\rho \in C^{1}\left(\left[t_{0}, \infty\right), \mathbb{R}\right)$ such that, for all sufficiently large $T_{*}$ and for $T>T_{*}$,

$$
\begin{aligned}
& \limsup _{t \rightarrow \infty} \int_{T}^{t}\left\{k \rho(s) \gamma(s) q(s) \phi\left(s, T_{*}\right)\right. \\
& \left.\quad-\frac{\alpha^{\alpha}}{(\alpha+1)^{\alpha+1}} \frac{c^{*} \gamma(s) r(s)\left(\rho^{\prime}(s)\right)^{\alpha+1}}{(\beta \rho(s))^{\alpha} R^{\beta-\alpha}\left(s, T_{*}\right)}\right\} d s \\
& \quad=\infty,
\end{aligned}
$$

with $c^{*}>0$, then any solution $x$ of (1) either is oscillatory or converges to zero as $t \rightarrow \infty$.

Proof. Let $x(t)$ be a nonoscillatory solution of (1). Without loss of generality, we may assume that there exists $t_{1} \geq t_{0}$ such that $x(t)>0, x(\tau(t))>0$, and $x(\delta(t))>0$ for $t \geq$ $t_{1}$. Proceeding as in the proof of Theorem 2, we see that (18) or (19) holds. If (18) holds, as in the proof of Theorem 2, we obtain (21), (24), (26), (28), and (34). Using (20), (24), (26), (28), and (34) in (21), we obtain

$$
\begin{aligned}
w^{\prime}(t) \leq & \frac{\rho_{+}^{\prime}(t)}{\rho(t)} w(t)-k \rho(t) \gamma(t) q(t) \phi\left(t, t_{2}\right) \\
& -\beta \rho(t) \gamma(t) r(t) \frac{\left(z^{\prime}(t)\right)^{\alpha+1}}{z^{\beta+1}(t)},
\end{aligned}
$$

for $t \geq t_{3}$. From (20), we have

$$
\begin{gathered}
\left(\frac{w(t)}{\rho(t) \gamma(t) r(t)}\right)^{(\alpha+1) / \alpha}=\left(\frac{\left(z^{\prime}(t)\right)^{\alpha}}{z^{\beta}(t)}\right)^{(\alpha+1) / \alpha} \\
=\frac{\left(z^{\prime}(t)\right)^{\alpha+1}}{z^{\beta(1+1 / \alpha)}(t)}=\frac{\left(z^{\prime}(t)\right)^{\alpha+1}}{z^{\beta+1}(t) z^{\beta / \alpha-1}(t)}
\end{gathered}
$$

that is,

$$
z^{\beta / \alpha-1}(t)\left(\frac{w(t)}{\rho(t) \gamma(t) r(t)}\right)^{(\alpha+1) / \alpha}=\frac{\left(z^{\prime}(t)\right)^{\alpha+1}}{z^{\beta+1}(t)} .
$$

Substituting (45) into (43) gives

$$
\begin{aligned}
w^{\prime}(t) \leq & \frac{\rho_{+}^{\prime}(t)}{\rho(t)} w(t)-k \rho(t) \gamma(t) q(t) \phi\left(t, t_{2}\right) \\
& -\frac{\beta z^{\beta / \alpha-1}(t)}{(\rho(t) \gamma(t) r(t))^{1 / \alpha}} w^{(\alpha+1) / \alpha}(t) .
\end{aligned}
$$

From (26) and the fact that $\beta / \alpha-1 \leq 0,(46)$ yields

$$
\begin{gathered}
w^{\prime}(t) \leq- \\
-k \rho(t) \gamma(t) q(t) \phi\left(t, t_{2}\right)+\frac{\rho_{+}^{\prime}(t)}{\rho(t)} w(t) \\
-\frac{c_{4} \beta R^{\beta / \alpha-1}\left(t, t_{2}\right)}{(\rho(t) \gamma(t) r(t))^{1 / \alpha}} w^{(\alpha+1) / \alpha}(t),
\end{gathered}
$$

where $c_{4}=c_{2}^{\beta / \alpha-1}$. Letting

$$
\begin{aligned}
& X=\left(\frac{c_{4} \beta R^{\beta / \alpha-1}\left(t, t_{2}\right)}{(\rho(t) \gamma(t) r(t))^{1 / \alpha}}\right)^{1 / \lambda} w(t), \quad \lambda=\frac{\alpha+1}{\alpha}, \\
& Y=\left\{\frac{1}{\lambda}\left(\frac{(\rho(t) \gamma(t) r(t))^{1 / \alpha}}{c_{4} \beta R^{\beta / \alpha-1}\left(t, t_{2}\right)}\right)^{1 / \lambda} \frac{\rho_{+}^{\prime}(t)}{\rho(t)}\right\}^{\alpha},
\end{aligned}
$$

in Lemma 1, (47) implies

$$
\begin{aligned}
w^{\prime}(t) \leq & -k \rho(t) \gamma(t) q(t) \phi\left(t, t_{2}\right) \\
& +\frac{\alpha^{\alpha}}{(\alpha+1)^{\alpha+1}} \frac{\gamma(t) r(t)\left(\rho_{+}^{\prime}(t)\right)^{\alpha+1}}{\left(c_{4} \beta \rho(t)\right)^{\alpha} R^{\beta-\alpha}\left(t, t_{2}\right)}
\end{aligned}
$$

for $t \geq t_{3}$.

Integrating the last inequality from $t_{3}$ to $t$ leads to

$$
\begin{aligned}
& \int_{t_{3}}^{t}\left\{k \rho(s) \gamma(s) q(s) \phi\left(s, t_{2}\right)\right. \\
& \left.\quad-\frac{\alpha^{\alpha}}{(\alpha+1)^{\alpha+1}} \frac{c_{3} \gamma(s) r(s)\left(\rho_{+}^{\prime}(s)\right)^{\alpha+1}}{(\beta \rho(s))^{\alpha} R^{\beta-\alpha}\left(s, t_{2}\right)}\right\} d s \\
& \quad \leq w\left(t_{3}\right)<\infty,
\end{aligned}
$$

which contradicts condition (42). 
Finally, if (19) holds, proceeding as in the proof of Theorem 2, we see that $\lim _{t \rightarrow \infty} x(t)=0$, which completes the proof of Theorem 3.

Theorem 4. Assume that (2) holds, and $\alpha \geq \beta \geq 1$. Suppose also that there exists a positive function $\rho \in C^{1}\left(\left[t_{0}, \infty\right), \mathbb{R}\right)$ such that, for all sufficiently large $T_{*}$ and for $T>T_{*}$,

$$
\begin{aligned}
\limsup _{t \rightarrow \infty} \int_{T}^{t}\left\{k \rho(s) \gamma(s) q(s) \phi\left(s, T_{*}\right)\right. \\
\left.-\frac{c^{*}(\gamma(s) r(s))^{1 / \alpha}\left(\rho_{+}^{\prime}(s)\right)^{2}}{4 \beta \rho(s) R^{\beta-1}\left(s, T_{*}\right)}\right\} d s=\infty,
\end{aligned}
$$

with $c^{*}>0$; then, any solution $x$ of (1) either oscillates or satisfies $x(t) \rightarrow 0$ as $t \rightarrow \infty$.

Proof. Let $x(t)$ be a nonoscillatory solution of (1). Without loss of generality, we may assume that there exists $t_{1} \geq t_{0}$ such that $x(t)>0, x(\tau(t))>0$, and $x(\delta(t))>0$ for $t \geq t_{1}$. Proceeding as in the proof of Theorems 2 and 3, we see that (18) or (19) holds. If (18) holds, as in the proof of Theorem 3, we obtain (47) which can be rewritten as

$$
\begin{aligned}
w^{\prime}(t) \leq & -k \rho(t) \gamma(t) q(t) \phi\left(t, t_{2}\right)+\frac{\rho_{+}^{\prime}(t)}{\rho(t)} w(t) \\
& -\frac{c_{4} \beta R^{\beta / \alpha-1}\left(t, t_{2}\right)}{(\rho(t) \gamma(t) r(t))^{1 / \alpha}} w^{1 / \alpha-1}(t) w^{2}(t),
\end{aligned}
$$

for $t \geq t_{3}$. From (20), we get

$$
\begin{aligned}
& w^{1 / \alpha-1}(t)=(\rho(t) \gamma(t) r(t))^{1 / \alpha-1}\left(\frac{\left(z^{\prime}(t)\right)^{\alpha}}{z^{\beta}(t)}\right)^{1 / \alpha-1} \\
& =(\rho(t) \gamma(t) r(t))^{1 / \alpha-1} \frac{\left(z^{\prime}(t)\right)^{1-\alpha}}{z^{\beta(1 / \alpha-1)}(t)} \\
& =(\rho(t) \gamma(t) r(t))^{1 / \alpha-1} \frac{z^{\beta-\beta / \alpha}(t)}{\left(z^{\prime}(t)\right)^{\alpha-1}} \\
& =(\rho(t) \gamma(t) r(t))^{1 / \alpha-1}\left(\frac{z(t)}{z^{\prime}(t)}\right)^{\alpha-1} z^{(\alpha-1)(\beta / \alpha-1)}(t) .
\end{aligned}
$$

From (24), we have

$$
\left(\frac{z(t)}{z^{\prime}(t)}\right)^{\alpha-1} \geq(\gamma(t) r(t))^{(\alpha-1) / \alpha} R^{\alpha-1}\left(t, t_{2}\right) .
$$

From (26) and the fact that $(\alpha-1)(\beta / \alpha-1) \leq 0$, we obtain

$$
z^{(\alpha-1)(\beta / \alpha-1)}(t) \geq c_{2}^{(\alpha-1)(\beta / \alpha-1)} R^{(\alpha-1)(\beta / \alpha-1)}\left(t, t_{2}\right) .
$$

Substituting (54) and (55) into (53) gives

$$
w^{1 / \alpha-1}(t) \geq \rho^{1 / \alpha-1}(t) c_{2}^{(\alpha-1)(\beta / \alpha-1)} R^{\beta-\beta / \alpha}\left(t, t_{2}\right) .
$$

Using (56) in (52), we obtain

$$
\begin{aligned}
w^{\prime}(t) \leq & -k \rho(t) \gamma(t) q(t) \phi\left(t, t_{2}\right)+\frac{\rho_{+}^{\prime}(t)}{\rho(t)} w(t) \\
& -\frac{\beta R^{\beta-1}\left(t, t_{2}\right)}{c_{3} \rho(t)(\gamma(t) r(t))^{1 / \alpha}} w^{2}(t) .
\end{aligned}
$$

Completing square with respect to $w$, it follows from (57) that

$$
\begin{aligned}
w^{\prime}(t) \leq & -k \rho(t) \gamma(t) q(t) \phi\left(t, t_{2}\right) \\
& +\frac{\mathcal{c}_{3}(\gamma(t) r(t))^{1 / \alpha}\left(\rho_{+}^{\prime}(t)\right)^{2}}{4 \beta \rho(t) R^{\beta-1}\left(t, t_{2}\right)} .
\end{aligned}
$$

Integrating the last inequality from $t_{3}$ to $t$ leads to

$$
\begin{aligned}
& \int_{t_{3}}^{t}\left\{k \rho(s) \gamma(s) q(s) \phi\left(s, t_{2}\right)\right. \\
& \left.-\frac{c_{3}(\gamma(s) r(s))^{1 / \alpha}\left(\rho_{+}^{\prime}(s)\right)^{2}}{4 \beta \rho(s) R^{\beta-1}\left(s, t_{2}\right)}\right\} d s \leq w\left(t_{3}\right) \\
& \quad<\infty,
\end{aligned}
$$

which contradicts condition (51).

Finally, if (19) holds, proceeding as in the proof of Theorem 2 , we see that $\lim _{t \rightarrow \infty} x(t)=0$, which completes the proof of Theorem 4 .

Remark 5. If $\alpha=\beta$, then we have $c^{*}=1$ in Theorems 2-4.

Example 6. Consider the neutral differential equation

$$
\begin{aligned}
(x(t) & \left.-\frac{1}{2} x\left(t-\frac{\pi}{2}\right)\right)^{\prime \prime} \\
& +\cos 4 t\left(x(t)-\frac{1}{2} x\left(t-\frac{\pi}{2}\right)\right)^{\prime} \\
& +(8+2 \sin 4 t) x\left(t+\frac{\pi}{2}\right)=0,
\end{aligned}
$$

for $t \geq 1$. Here, we have $r(t)=1, h(t)=\cos 4 t, q(t)=8+$ $2 \sin 4 t, \tau(t)=t-\pi / 2, \delta(t)=t+\pi / 2, f(u)=u$, and $\alpha=\beta=1$. Then,

$$
\begin{aligned}
\gamma(t) & =\exp \left(\int_{1}^{t} \cos 4 s d s\right) \\
& =e^{(1 / 4) \sin 4 t-(1 / 4) \sin 4}, \\
\int_{1}^{t} \frac{d s}{(\gamma(s) r(s))^{1 / \alpha}} & =e^{(1 / 4) \sin 4} \int_{1}^{t} \frac{d s}{e^{(1 / 4) \sin 4 s}} \\
& \geq \frac{e^{(1 / 4) \sin 4}}{e^{1 / 4}} \int_{1}^{t} d s \longrightarrow \infty
\end{aligned}
$$


so (2) holds. Since

$$
\begin{aligned}
\phi\left(t, T_{*}\right) & =\phi(t, 1)=1, \\
R^{\beta}\left(t, T_{*}\right) & =R^{\beta}(t, 1)=e^{(1 / 4) \sin 4} \int_{1}^{t} \frac{d s}{e^{(1 / 4) \sin 4 s}} \\
& \geq \frac{e^{(1 / 4) \sin 4}}{e^{1 / 4}} \int_{1}^{t} d s,
\end{aligned}
$$

condition (7) with $\rho(t)=t$ and $T>T_{*}=1$ becomes

$$
\begin{aligned}
& \limsup _{t \rightarrow \infty} \int_{T}^{t}\left\{k \rho(s) \gamma(s) q(s) \phi\left(s, T_{*}\right)\right. \\
& \left.-\frac{c^{*} \rho_{+}^{\prime}(s)}{R^{\beta}\left(s, T_{*}\right)}\right\} d s \\
& \geq \limsup _{t \rightarrow \infty} \int_{T}^{t}\left\{s e^{(1 / 4) \sin 4 s-(1 / 4) \sin 4}(8+2 \sin 4 s)\right. \\
& \left.-\frac{e^{-(1 / 4) \sin 4} e^{1 / 4}}{(s-1)}\right\} d s \geq e^{-(1 / 4) \sin 4} \\
& \cdot \limsup _{t \rightarrow \infty} \int_{T}^{t}\left\{6 s e^{-1 / 4}-\frac{e^{1 / 4}}{(s-1)}\right\} d s=e^{-(1 / 4) \sin 4} \\
& .\left.\limsup _{t \rightarrow \infty}\left(3 e^{-1 / 4} s^{2}-e^{1 / 4} \ln (s-1)\right)\right|_{T} ^{t}=\infty ;
\end{aligned}
$$

that is, condition (7) holds. So every solution of (60) either is oscillatory or satisfies $x(t) \rightarrow 0$ as $t \rightarrow \infty$ by Theorem 2 . In fact, it is easy to see that one oscillatory solution of $(60)$ is $x(t)=\cos 4 t$.

Example 7. The neutral differential equation

$$
\begin{aligned}
\left(t^{3}\left(z^{\prime}(t)\right)^{5}\right)^{\prime}+t^{2}\left(z^{\prime}(t)\right)^{5}+(\sqrt[5]{t}-1)^{3} x^{3}\left(\frac{t}{2}\right) & =0 \\
t & \geq 1
\end{aligned}
$$

is a special case of (1) with $z(t)=x(t)-(1 / 3) x(t-\pi / 4)$, $r(t)=t^{3}, h(t)=t^{2}, q(t)=(\sqrt[5]{t}-1)^{3}, \tau(t)=t-\pi / 4, \delta(t)=t / 2$, $f(u)=u^{3}, \alpha=5$, and $\beta=3$. Clearly,

$$
\begin{aligned}
\gamma(t) & =\exp \left(\int_{1}^{t} \frac{1}{s} d s\right)=e^{\ln t-\ln 1}=t, \\
\int_{1}^{\infty} \frac{d s}{(\gamma(s) r(s))^{1 / \alpha}} & =\int_{1}^{\infty} \frac{d s}{s^{4 / 5}}=\infty,
\end{aligned}
$$

so (2) holds. Since

$$
\begin{aligned}
\phi\left(t, T_{*}\right) & =\phi(t, 1)=\left(\frac{\int_{1}^{t / 2}\left(d s / s^{4 / 5}\right)}{\int_{1}^{t}\left(d s / s^{4 / 5}\right)}\right)^{3} \\
& =\frac{1}{2^{3 / 5}} \frac{(\sqrt[5]{t}-\sqrt[5]{2})^{3}}{(\sqrt[5]{t}-1)^{3}}
\end{aligned}
$$

condition (42) with $\rho(t)=1$ and $T>T_{*}=1$ becomes

$$
\begin{aligned}
\limsup _{t \rightarrow \infty} \int_{T}^{t} s \frac{1}{2^{3 / 5}}(\sqrt[5]{s}-\sqrt[5]{2})^{3} d s \\
\quad=\frac{1}{2^{3 / 5}} \limsup _{t \rightarrow \infty} \int_{T}^{t} s(\sqrt[5]{s}-\sqrt[5]{2})^{3} d s=\infty ;
\end{aligned}
$$

that is, (42) holds. Therefore, by Theorem 3, a solution of (64) either is oscillatory or converges to zero.

\section{Competing Interests}

The authors declare that there are no competing interests regarding the publication of this paper.

\section{References}

[1] B. Sturm, "Sur les équation différentielles linéaires du second ordré," Journal de Mathématiques Pures et Appliquées, vol. 1, pp. 106-186, 1836.

[2] Ch. G. Philos, "Oscillation theorems for linear differential equations of second order," Archiv der Mathematik, vol. 53, no. 5, pp. 482-492, 1989.

[3] I. V. Kamenev, "An integral criterion for oscillation of linear differential equations of second order," Mathematical notes of the Academy of Sciences of the USSR, vol. 23, no. 2, pp. 136-138, 1978.

[4] J. S. W. Wong, "Necessary and sufficient conditions for oscillation of second order neutral differential equations," Journal of Mathematical Analysis and Applications, vol. 252, no. 1, pp. 342$352,2000$.

[5] L. Erbe, T. S. Hassan, and A. Peterson, "Oscillation criteria for nonlinear functional neutral dynamic equations on time scales," Journal of Difference Equations and Applications, vol. 15, no. 1112, pp. 1097-1116, 2009.

[6] J. R. Graef, M. K. Grammatikopoulos, and P. W. Spikes, "On the asymptotic behavior of solutions of a second order nonlinear neutral delay differential equation," Journal of Mathematical Analysis and Applications, vol. 156, no. 1, pp. 23-39, 1991.

[7] J. R. Graef, R. Savithri, and E. Thandapani, "Oscillation of first order neutral delay differential equations," Electronic Journal of Qualitative Theory of Differential Equations, no. 12, pp. 1-11, 2003.

[8] J. R. Graef, R. Savithri, and E. Thandapani, "Oscillatory properties of third order neutral delay differential equations," in Proceedings of the 4th International Conference on Dynamical Systems and Differential Equations, pp. 342-350, Wilmington, NC, USA, May 2002.

[9] J. R. Graef and E. Tunç, "Oscillation criteria for second order functional dynamic equations on time-scales," International Electronic Journal of Pure and Applied Mathematic, vol. 8, no. 1, pp. 17-31, 2014.

[10] E. Tunç, "Oscillation results for even order trinomial functional differential equations with damping," Applications and Applied Mathematics, vol. 9, no. 2, pp. 696-717, 2014.

[11] E. Tunç and J. R. Graef, "Oscillation results for second order neutral dynamic equatitons with distributed deviating arguments," Dynamic Systems and Applications, vol. 23, pp. 289-303, 2014. 
[12] E. Tunc, "Oscillation results for even order functional dynamic equations on time scales," Electronic Journal of Qualitative Theory of Differential Equations, no. 27, pp. 1-14, 2014.

[13] B. Baculíková and J. Džurina, "Oscillation of third-order neutral differential equations," Mathematical and Computer Modelling, vol. 52, no. 1-2, pp. 215-226, 2010.

[14] B. Baculíková and J. Džurina, "Oscillation theorems for secondorder nonlinear neutral differential equations," Computers \& Mathematics with Applications, vol. 62, no. 12, pp. 4472-4478, 2011.

[15] Y. V. Rogovchenko and F. Tuncay, "Oscillation criteria for second-order nonlinear differential equations with damping," Nonlinear Analysis: Theory, Methods \& Applications, vol. 69, no. 1, pp. 208-221, 2008.

[16] T. Li and Yu. V. Rogovchenko, "Oscillation theorems for secondorder nonlinear neutral delay differential equations," Abstract and Applied Analysis, vol. 2014, Article ID 594190, 5 pages, 2014.

[17] T. Li and Y. V. Rogovchenko, "Oscillatory behavior of secondorder nonlinear neutral differential equations," Abstract and Applied Analysis, vol. 2014, Article ID 143614, 8 pages, 2014.

[18] T. Li, Y. V. Rogovchenko, and C. Zhang, "Oscillation results for second-order nonlinear neutral differential equations," Advances in Difference Equations, vol. 2013, article 336, pp. 1-13, 2013.

[19] T. Li, E. Thandapani, J. R. Graef, and E. Tunc, "Oscillation of second-order Emden-Fowler neutral differential equations," Nonlinear Studies, vol. 20, no. 1, pp. 1-8, 2013.

[20] Q. Li, R. Wang, F. Chen, and T. Li, "Oscillation of second-order nonlinear delay differential equations with nonpositive neutral coefficients," Advances in Difference Equations, vol. 2015, no. 1, article 35, pp. 1-7, 2015.

[21] S. R. Grace, J. R. Graef, S. Panigrahi, and E. Tunc, "On the oscillatory behavior of even order neutral delay dynamic equations on time-scales," Electronic Journal of Qualitative Theory of Differential Equations, vol. 2012, no. 96, pp. 1-12, 2012.

[22] S. R. Grace, "On the oscillation of higher order dynamic equations," Journal of Advanced Research, vol. 4, no. 2, pp. 201204, 2013.

[23] S. R. Grace, "On the oscillation of $n$th order dynamic equations on time-scales," Mediterranean Journal of Mathematics, vol. 10, no. 1, pp. 147-156, 2013.

[24] Q. Yang, L. Yang, and S. Zhu, "Interval criteria for oscillation of second-order nonlinear neutral differential equations," Computers \& Mathematics with Applications, vol. 46, no. 5-6, pp. 903-918, 2003.

[25] H. Qin, N. Shang, and Y. Lu, "A note on oscillation criteria of second order nonlinear neutral delay differential equations," Computers \& Mathematics with Applications, vol. 56, no. 12, pp. 2987-2992, 2008.

[26] H. Guan and Y. Yu, "Oscillation of second order quasi-linear neutral delay differential equations," International Journal of Mathematics and its Applications, vol. 3, no. 2, pp. 17-24, 2015.

[27] M. Bohner, S. R. Grace, I. Sağer, and E. Tunç, "Oscillation of third-order nonlinear damped delay differential equations," Applied Mathematics and Computation, vol. 278, pp. 21-32, 2016.

[28] M. Sheikholeslami, D. D. Ganji, H. R. Ashorynejad, and H. B. Rokni, "Analytical investigation of Jeffery-Hamel flow with high magnetic field and nanoparticle by Adomian decomposition method," Applied Mathematics and Mechanics, vol. 33, no. 1, pp. 25-36, 2012.
[29] M. Sheikholeslami and D. D. Ganji, "Magnetohydrodynamic flow in a permeable channel filled with nanofluid," Scientia Iranica B, vol. 21, no. 1, pp. 203-212, 2014.

[30] M. Shekholeslami, H. R. Ashorynejad, D. Domairry, and I. Hashim, "Investigation of the laminar viscous flow in a semiporous channel in the presence of uniform magnetic field using optimal homotopy asymptotic method," Sains Malaysiana, vol. 41, no. 10, pp. 1281-1285, 2012.

[31] M. Sheikholeslami and D. D. Ganji, "Heat transfer of Cu-water nanofluid flow between parallel plates," Powder Technology, vol. 235, pp. 873-879, 2013.

[32] M. Sheikholeslami, H. R. Ashorynejad, D. D. Ganji, and A. Yıldirım, "Homotopy perturbation method for threedimensional problem of condensation film on inclined rotating disk," Scientia Iranica, vol. 19, no. 3, pp. 437-442, 2012.

[33] M. Sheikholeslami and D. D. Ganji, "Nanofluid flow and heat transfer between parallel plates considering Brownian motion using DTM," Computer Methods in Applied Mechanics and Engineering, vol. 283, pp. 651-663, 2015.

[34] J. K. Hale, Theory of Functional Differential Equations, Springer, New York, NY, USA, 2nd edition, 1977.

[35] G. H. Hardy, J. E. Littlewood, and G. Polya, Inequalities, Reprint of the 1952 edition, Cambridge University Press, Cambridge, UK, 1988. 


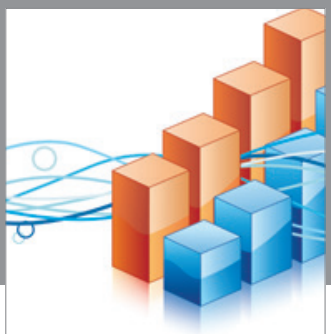

Advances in

Operations Research

vatem alat4

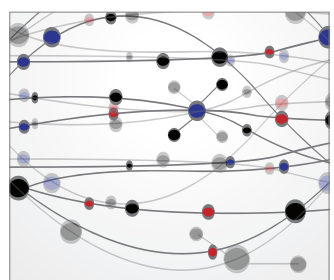

\section{The Scientific} World Journal
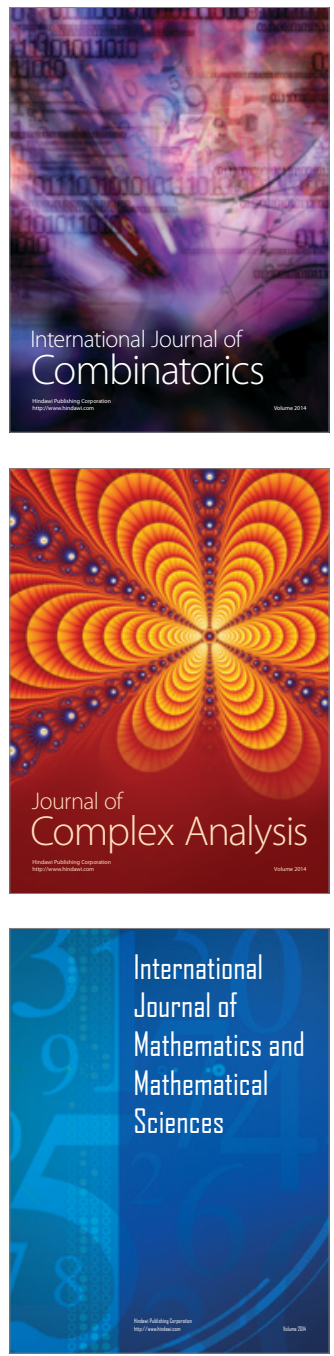
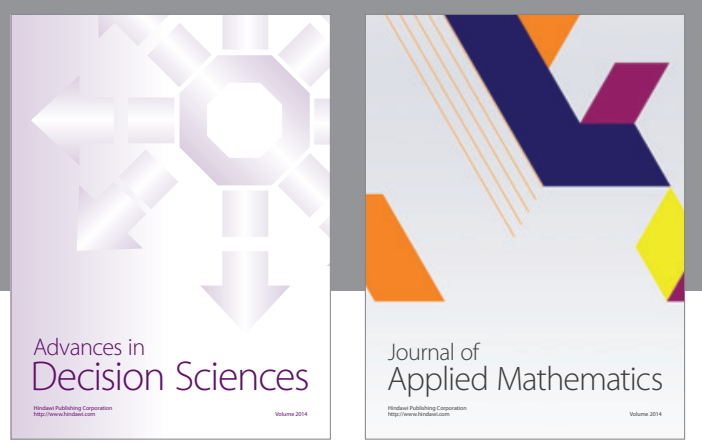

Algebra

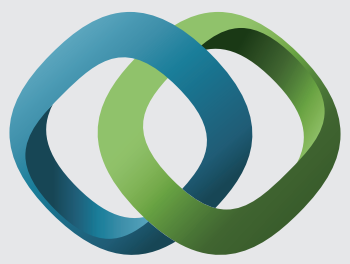

\section{Hindawi}

Submit your manuscripts at

http://www.hindawi.com
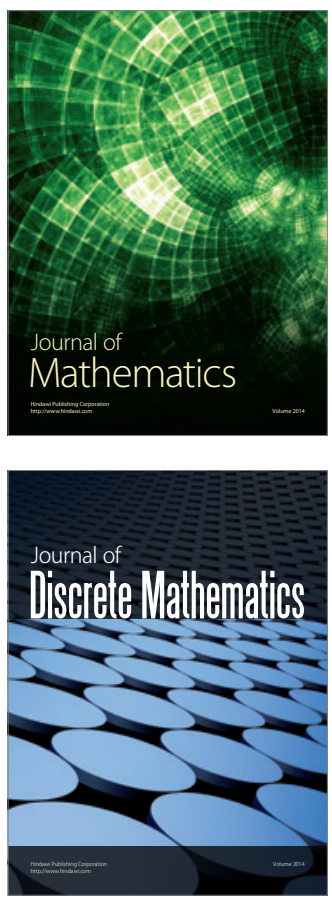

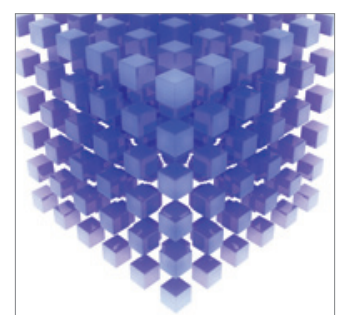

Mathematical Problems in Engineering
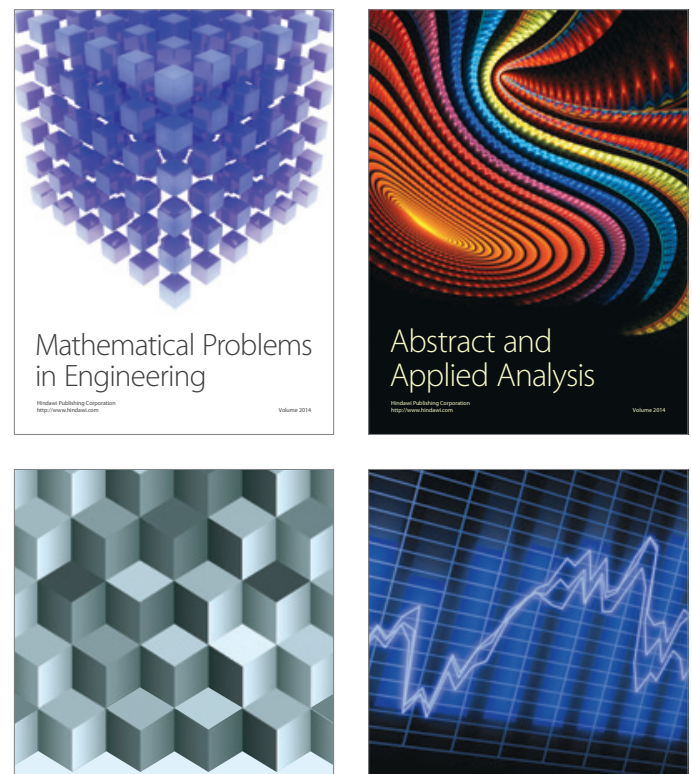

Journal of

Function Spaces

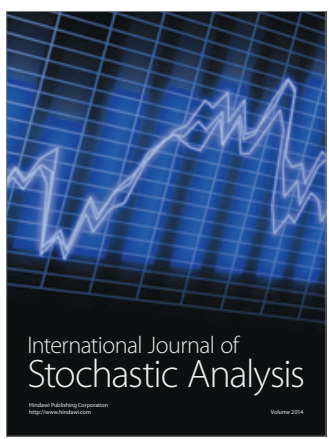

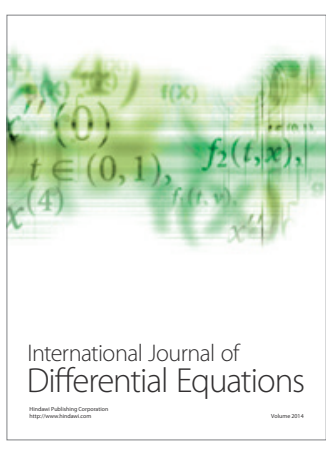
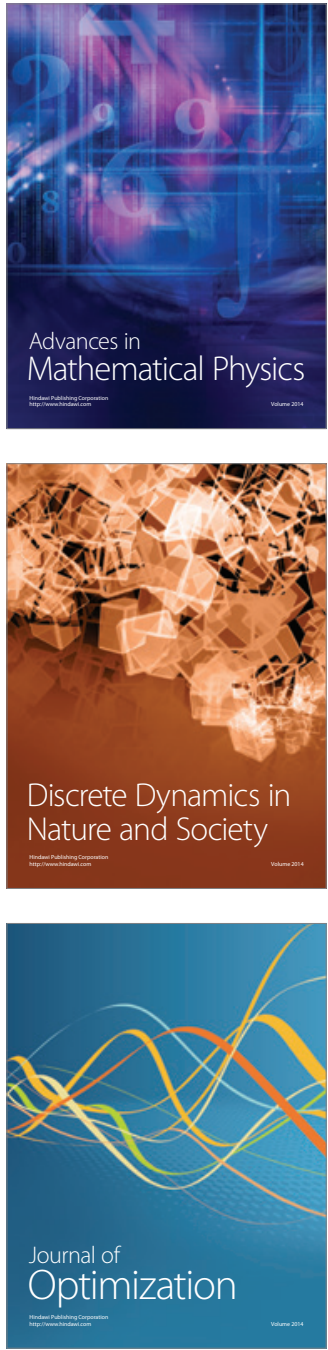